\title{
Automated image analysis to improve bead ingestion toxicity test counts in the protozoan Tetrahymena pyriformis
}

\author{
N. Dias $^{1}$, A.L. Amaral ${ }^{1,2}$, E.C. Ferreira ${ }^{1}$ and N. Lima ${ }^{1}$ \\ ${ }^{1}$ Centro de Engenharia Biológica, Universidade do Minho, Campus de Gualtar, Braga, Portugal, and ${ }^{2}$ Departamento de Tecnologia \\ Química, Escola Superior de Tecnologia e de Gestão, Instituto Politécnico de Bragança, Campus de Santa Apolónia, Bragança, \\ Portugal
}

2003/30: received 14 January 2003, revised 24 March 2003 and accepted 1 June 2003

\begin{abstract}
N. DIAS, A.L. AMARAL, E.C. FERREIRA AND N. LIMA. 2003.
\end{abstract}

Aims: To improve bead ingestion counts in Tetrahymena pyriformis by automated image analysis as an alternative to direct-counts.

Methods and Results: Fluorescent latex beads were added to T. pyriformis cultures for ingestion tests. The number of beads ingested by 25 cells was counted directly by epifluorescence microscopy and compared with similar data from image analysis. ANOVA indicated that counts were not significantly different $(P<0 \cdot 05)$. The image analysis particularly provided advantages in terms of speed.

Conclusions: The image analysis is superior to direct beads counting in T. pyriformis particularly in terms of speed of analysis.

Significance and Impact of the Study: The image analysis method is very rapid and will allow many more toxicological analyses to be undertaken with less operator error.

Keywords: Tetrahymena pyriformis, image analysis, ingestion, toxicity tests, bead counts.

\section{INTRODUCTION}

The protozoan Tetrahymena is used to assess the bioactivity of various chemicals because of its ability to ingest model substances and can be used as an alternative to laboratory animals for this purpose (Nilsson 1981; Wakatsuki et al. 1986; Stefanidou et al. 1990; Alevisopoulos et al. 1997; Stefanidou et al. 1999; Schiess et al. 2001). Nicolau et al. (1999) studied the influence of several toxicants on Tetrahymena pyriformis grazing activity using fluorescent labelled latex beads (FLLB), which are considered a valid material for these purposes. Recently, green fluorescent proteinexpressing Escherichia coli coupled with fluorometry has been used to determine protozoan ingestion rates (Parry et al. 2001). Image analysis techniques provide qualitative and quantitative analysis of micro-organisms and provide

Correspondence to: Nelson Lima, Centro de Engenharia Biológica, Universidade do Minho, Campus de Gualtar,4710-057,Braga, Portugal(e-mail: nelson@iec.um inho.pt). significant advantages over microscopic visualization using the naked eye (e.g. speed, accuracy and objectivity) (Buño et al. 1998). The methodology has been used in the (a) determination of bacterial abundance, biovolume, morphology and growth (Blackburn et al. 1998); (b) detection of confluent microbial colonies for automated counting (Corkidi et al. 1998); (c) analysis of biofilms (Kuehn et al. 1998) and (d) microbial community characterization (Amaral et al. 1999). In the present work, image analysis was compared with direct FLLB counting to assess ingestion by $T$. pyriformis.

\section{MATERIALS AND METHODS}

\section{Micro-organism and culture conditions}

Tetrahymena pyriformis (strain GL, ref. CCAP/1630/1F; Culture Collection of Algae and Protozoa, UK) was grown axenically to a density of approximately $10^{4}$ cells $\mathrm{ml}^{-1}$ in the exponential phase, at room temperature $\left(c a 22^{\circ} \mathrm{C}\right), \mathrm{pH}$

(C) 2003 The Society for Applied Microbiology

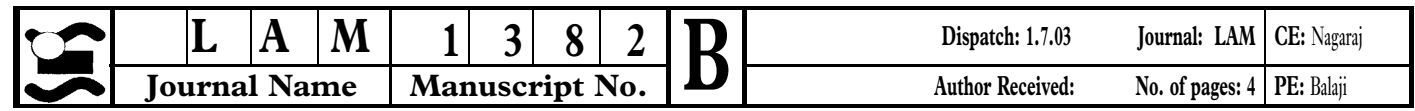


7.0-7·5. Proteose peptone yeast extract medium (PPY), Difco proteose peptone $2 \%$ and Oxoid yeast extract $0.25 \%$, was used for growth. The medium was filtered twice

1 ( $0.45 \mu \mathrm{m}$ membrane; Gelman $)$, before autoclave sterilization, to avoid particle background upon microscopic 5 observation.

\section{Ingestion assay}

The assay was performed in 2-ml Eppendorf test tubes, by adding the yellow-green FluoSpheres ${ }^{\circledR}$ fluorescent microspheres $(0.5 \mu \mathrm{m}$ diameter, Molecular Probes Europe BV) to the cells at a rate of $1.05 \times 10^{6}$ bead per ml (Nicolau et al. 1999). The solution was vortexed for a minimum of $5 \mathrm{~min}$ to avoid bead clustering. After incubation in the dark for $20 \mathrm{~min}$, samples were fixed for $1 \mathrm{~h}$ with neutral buffered formalin $[10 \%(\mathrm{v} / \mathrm{v})$ formalin in phosphatebuffered saline (PBS) $\mathrm{pH} 7 \cdot 0$, at a final concentration of $2-5 \%(\mathrm{v} / \mathrm{v})]$. Cells were washed and resuspended in fresh $0 \cdot 1 \%(\mathrm{w} / \mathrm{v})$ sodium azide in PBS and stored in the dark for subsequent analysis. Bead counting was performed with 3 an epifluorescence Laborlux S (Leitz, Wetzlar) microscope using UV light (EX 450-490 nm excitation filter). The number of beads ingested by 25 randomly selected cells was counted at $1000 \times$ magnification, with minimum field diaphragm aperture in order to minimize excess illumination, control glare and avoid internal reflection. Samples from each independent assay were taken at $0 \cdot 1,1$ and $24 \mathrm{~h}$.

\section{Image acquisition}

The image acquisition (50 images per sample) was accomplished through fluorescence microscopy using the same conditions as described above. All the images were digitized using a 3CCD DXC-9100P colour camera (Sony, Tokyo, Japan) and a Matrox Meteor II frame grabber (Matrox,

4Dorval), with a $768 \times 576$ pixel size and 24 bits per image resolution ( 8 bits per each colour channel, red, green and blue) by the Matrox Intellicam (Matrox) software package. Image processing and analysis was then performed in MATLAB 5.1 software package (The Mathworks Inc., Natick, MA, USA). Graphical analysis of data from MATLAB was performed with EXCEL ${ }^{\mathrm{TM}}$ (Microsoft Corp., Redmond, DC, USA).

\section{Image processing and analysis}

With the purpose of determining the total area of beads present in each image, a program was created in MATLAB comprising the following steps:

i Image loading: the images were loaded from a folder and acquired as colour TIFF format. ii Channel selection: the image was then decomposed in its three colour channels and the green channel image was subsequently used in the program.

iii Image smoothing: the resulting image was smoothed by a $2 \times 2$ window Wiener filter which performs a twodimensional adaptive noise-removal filtering using the Wiener method based on statistics estimated from the local neighbourhood of each pixel. This filter first estimates the local mean $\mu$ and the local variance $\sigma^{2}(1)$, (2):

$$
\begin{gathered}
\mu=\frac{1}{N M} \sum_{n_{1}, n_{2} \in \eta} a\left(n_{1}, n_{2}\right) \\
\sigma^{2}=\frac{1}{N M} \sum_{n_{1}, n_{2} \in \eta} a^{2}\left(n_{1}, n_{2}\right)-\mu^{2}
\end{gathered}
$$

where $\eta$ is the $N \times M$ local neighbourhood of each pixel in the image $a$. The filter then creates a pixelwise Wiener filter $b$ using these estimates (3):

$$
b\left(n_{1}, n_{2}\right)=\mu+\frac{\sigma^{2}-v^{2}}{\sigma^{2}} \times\left[a\left(n_{1}, n_{2}\right)-\mu\right]
$$

where $v^{2}$ is the noise variance, i.e. the average of all the local estimated variances.

iv Segmentation: the resulting image of the previous step was then segmented into a binary image with pixels value of 1 for the objects and 0 for the background. Hence, the pixels above a user chosen threshold (default value of 0.35 ) were segmented to 1 (beads) and the values below that threshold to 0 (background).

v Debris removal: in this step, all the objects in the image with an area smaller than 10 pixels were considered as debris and, therefore, removed.

vi Parameters determination: the morphological parameter beads area was then determined from the final binary image, as the projected surface of the beads, i.e. the total number of pixels belonging to all beads present in the image.

vii Result saving: the final binary image was saved in a TIFF format and the beads area in an ASCII file.

\section{Bead number determination}

A previous calibration of 50 beads was performed in order to convert the pixel area of one bead given by image analysis, in the numerical determination of fluorescent beads. The mean area $A$ of a single bead was calculated by:

$$
A=\pi r^{2}
$$

where $r$ is the radius of the bead.

For the $1000 \times$ magnification, the area of each pixel was $0.01918 \mu \mathrm{m}^{2}$ and the average bead area was found to be $0 \cdot 19625 \mu \mathrm{m}^{2}$ corresponding to $10 \cdot 232$ pixels. 


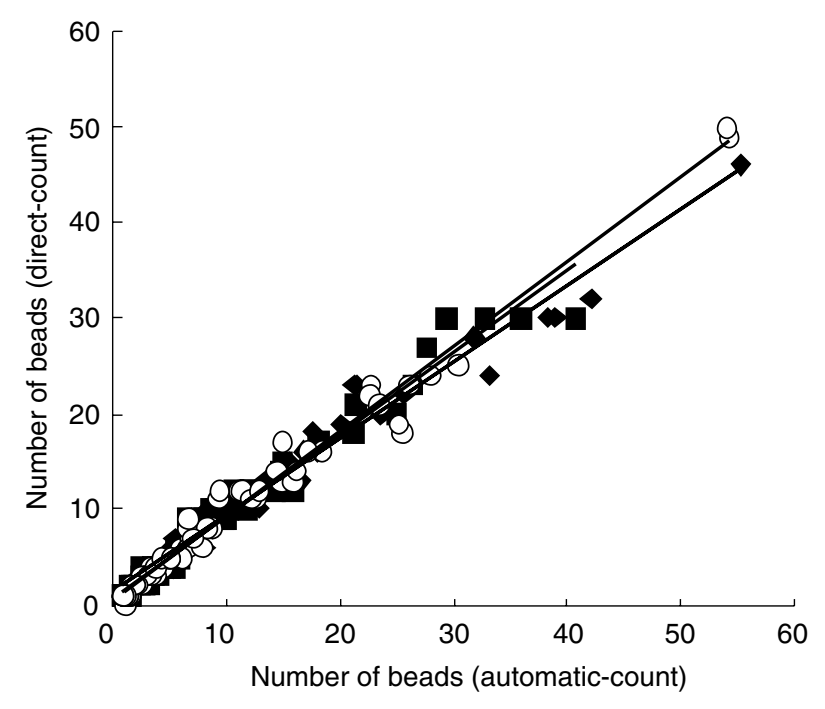

Fig. 1 Regression lines between the number of beads counted by direct count and automatic count, in 25 protozoa, at different sampling times $(\diamond) 0 \cdot 1 \mathrm{~h},(\boldsymbol{\square}) 1 \mathrm{~h}$ and $(\bigcirc) 24 \mathrm{~h}$

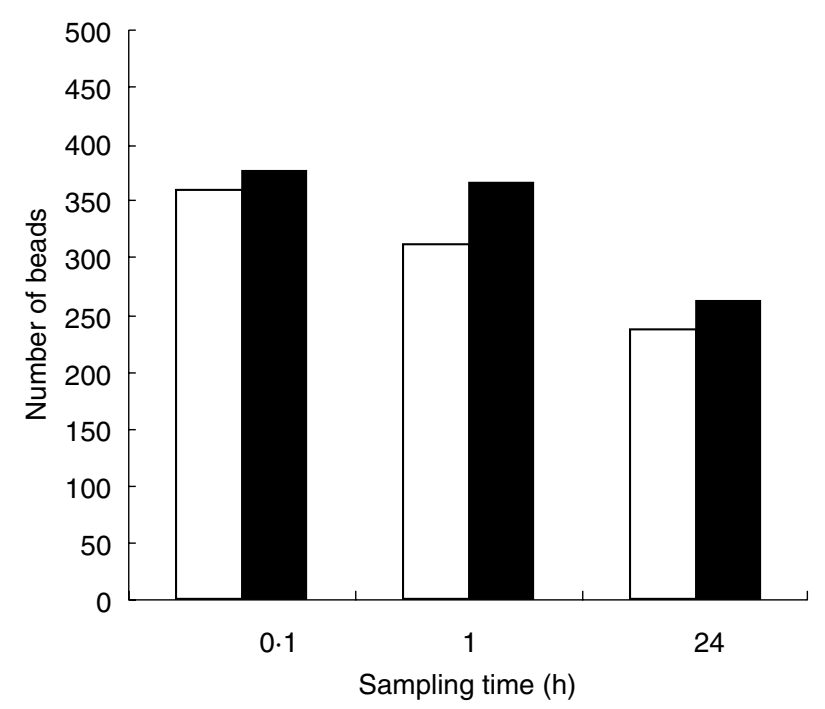

Fig. 2 Enumeration of beads ingested by Tetrahymena pyriformis, in 25 protozoa counted by direct count $(\square)$ and by automatic count ( $(\mathbf{\square})$ at different sampling times

\section{Statistical data analysis}

ANOVA was used to test the statistical significance $(P<0.05)$ of differences between automatic and direct count data.

\section{RESULTS}

Prior to the bead number determination, a microsphere size calibration must be performed. The area of 50 isolated beads was determined and a mean area value of $9.958 \pm 2.030 \mu \mathrm{m}^{2}$ was obtained. After image acquisition, the segmentation was achieved using a chosen threshold or the default value of $0 \cdot 35$.

At the sampling times $0 \cdot 1,1$ and $24 \mathrm{~h}$ good correlations of $0.974,0.971$ and 0.983 , respectively, were obtained for the two methods. The linear regressions between the automatic and direct-count are shown in Fig. 1. Some variation was observed in samples where the number of beads ingested by $T$. pyriformis was greater than 20 . No significant differences $(P<0.05)$ were found between the numbers of beads counted by both methods at any time of incubation (Fig. 2).

\section{DISCUSSION}

Image analysis is used routinely in cellular biology studies. The present work demonstrates that the automated count method was as effective as the conventional method using a microscope with the naked eye. The computerized method allowed the acquisition of hundreds of images and subsequent analysis in $1 \mathrm{~h}$ compared with approximately three to four samples by conventional method. The choice of the threshold value was of crucial importance in order to resolve the beads from background and therefore great care is required with this step.

Some underestimation in the number of beads counted by the direct-count method occurred if more than 20 beads were ingested because of the intense fluorescence of the beads. This is not the case with the automated method. Pläsier $e t$ al. (1999) refer to the intrinsic subjectiveness of the direct-count method. Accordingly, the counting of a large number of beads in this work may differ from person to person or even within different counts by the same operator. In conclusion, the high correlation values and the absence of significant differences between each method at all sampling times suggest the superiority of image analysis to quantify beads inside protozoa in subsequent toxicological studies.

\section{ACKNOWLEDGEMENTS}

The authors wish to thank Dr Russell Paterson for his helpful comments and corrections. N. Dias and A. L. Amaral were supported by PRAXIS-XXI BD/20328/99 and BD/20325/99 grants, respectively, from FCT (Fundação para a Ciência e Tecnologia), Portugal.

\section{REFERENCES}

Alevisopoulos, G., Stefanidou, M., Pagou, M., Siatra-Papastaikoudi, T. and Koutselinis, A. (1997) The effects of two new amphetaminelike derivatives on the phagocytic ability of the protozoon Tetrahymena pyriformis. Alternatives to Laboratory Animals 25, 547-553.

Amaral, A.L., Baptiste, C., Pons, M.N., Nicolau, A., Lima, N., Ferreira, E.C., Mota, M. and Vivier, H. (1999) Semi-automated 
recognition of protozoa by image analysis. Biotechnology Techniques 13, 111-118.

Blackburn, N., Hagstrom, A., Wikner, J., Cuadros-Hansson, R. and Bjornsen, P.K. (1998) Rapid determination of bacterial abundance, biovolume, morphology, and growth by neural network-based image analysis. Applied and Environmental Microbiology 64, 3246-3255.

Buño, I., Juarranz, A., Cañete, M., Villanueva, A., Gosalvez, J. and Stockert, J.C. (1998) Image processing and analysis of fluorescent labelled cytoskeleton. Micron 29, 445-449.

Corkidi, G., Diaz-Uribe, R., Folch-Mallol, J.L. and Nieto-Sotelo, J. (1998) COVASIAM: an image analysis method that allows detection of confluent microbial colonies and colonies of various sizes for automated counting. Applied and Environmental Microbiology 64, $1400-1404$.

Kuehn, M., Hausner, M., Bungartz, H.J., Wagner, M., Wilderer, P.A. and Wuertz, S. (1998) Automated confocal laser scanning microscopy and semiautomated image processing for analysis of biofilms. Applied and Environmental Microbiology 64, 4115-4127.

Nicolau, A., Mota, M. and Lima, N. (1999) Physiological responses of Tetrahymena pyriformis to copper, zinc, cyclohexymide and Triton X-100. FEMS Microbiology Ecology 30, 209-216.

Nilsson, J.R. (1981) Effects of copper on phagocytosis in Tetrahymena. Protoplasma 109, 359-370.
Parry, J.D., Heaton, K., Drinkall, J. and Jones, H.L.J. (2001) Feasibility of using GFP-expressing Escherichia coli, coupled with fluorometry, to determine protozoan ingestion rates. FEMS Microbiology Ecology 35, 11-17.

Pläsier, B., Lloyd, D.R., Paul, G.C., Thomas, C.R. and Al-Rubeai, M. (1999) Automatic image analysis for quantitation of apoptosis in animal cell culture by annexin $\mathrm{V}$ affinity assay. Fournal of Immunological Methods 229, 81-95.

Schiess, N., Csaba, G. and Kohidai, L. (2001) Chemotactic selection with insulin, di-iodotyrosine and histamine alters the phagocytic responsiveness of Tetrahymena. Comparative Biochemistry and Physiology 128, 521-530.

Stefanidou, M.E., Georgiou, M., Maravelias, C. and Koutselinis, A. (1990) The effects of morphine, cocaine, amphetamine and hashish on the phagocytosis of the protozoon Tetrahymena pyriformis strain W. Toxicology in Vitro 4, 779-781.

Stefanidou, M.E., Alevisopoulos, G., Maravelias, C., Loutsidis, C. and Koutselinis, A. (1999) Phagocytosis of the protozoon Tetrahymena pyriformis as an endpoint in the estimation of cocaine salt and cocaine freebase toxicity. Addiction Biology 4, 449-452.

Wakatsuki, T., Tazaki, Y. and Imahara, H. (1986) Respiratory inhibition by copper in Tetrahymena pyriformis GL. Fournal of Fermentation Technology 64, 119-127. 